\title{
On the Composition Ideals of Schatten Class Type Mappings
}

\author{
Abdelaziz Belaada, Khalil Saadi, and Abdelmoumen Tiaiba \\ Laboratoire d'Analyse Fonctionnelle et Géométrie des Espaces, M’sila University, BP 166, Ichbilia, 28003 M'sila, Algeria \\ Correspondence should be addressed to Khalil Saadi; kh_saadi@yahoo.fr
}

Received 23 June 2016; Accepted 25 July 2016

Academic Editor: Alfred Peris

Copyright (C) 2016 Abdelaziz Belaada et al. This is an open access article distributed under the Creative Commons Attribution License, which permits unrestricted use, distribution, and reproduction in any medium, provided the original work is properly cited.

We study the composition ideals of multilinear and polynomial mappings generated by Schatten classes. We give some coincidence theorems for Cohen strongly 2-summing multilinear operators and factorization results like that given by Lindenstrauss-Pełczński for Hilbert Schmidt linear operators.

\section{Introduction and Preliminaries}

Let $H, K$ be two Hilbert spaces and $\mathcal{S}_{p}(K ; H)$ the $p$ th Schatten class, that is, the space of compact operators $u: K \rightarrow$ $H$ such that $\operatorname{tr}\left(|u|^{p}\right)<\infty$. This space is an ideal of linear operators and coincides with the space of Hilbert Schmidt operators for $p=2$. Pietsch in [1] has introduced and studied multilinear ideals, by introducing two methods to construct multilinear ideals from a given linear ideal. It is well known that some classes of multilinear operators can be interpreted via these methods of Pietsch, namely, the classes of $p$-dominated, weakly compact, and compact multilinear operators. If we consider the Schatten class $\mathcal{S}_{p}$, Braunss and Junek [2] have studied the multi-ideals generated by this linear ideal, where the authors have used the factorization method of Pietsch. Andrade Mendes [3] has given some factorization results of these mappings and showed that the resulting space by this procedure $\mathscr{L}\left(\mathcal{S}_{2}\right)$ coincides with the class of 2-dominated multilinear operators. The results in this paper are concentrated to study the multilinear operators generated by the Schatten class. We give another class of $m$ linear mappings, noted by $\mathcal{S}_{p} \circ \mathscr{L}$, whose operators $T$ can be written as $T=u \circ A$ with $u$ belonging to $\mathcal{S}_{p}$ and $A$ is a multilinear operator (this technique of factorization known as composition ideals; see [4]). We will show that the class $\delta_{2} \circ \mathscr{L}$ coincides with $\mathscr{D}_{2}^{m}$, the class of Cohen strongly 2summing multilinear operators. We also present some factorization results for multilinear mappings of type $\mathcal{S}_{2} \circ \mathscr{L}$ like that given by Lindenstrauss-Pełczński for Hilbert Schmidt linear operators and Mendes for multilinear mappings of type $\mathscr{L}\left(\mathcal{S}_{p}\right)$. The same results are given for the polynomial case.

This paper is organized as follows. In the rest of Section 1, we recall some standard notations and definitions concerning polynomial and multilinear mappings. Section 2 is devoted to prove our main results; we study the class $\mathcal{S}_{p} \circ \mathscr{L}$ of multilinear operators $T$ which admit a factorization $T=$ $u \circ A$ where $u$ is in $\delta_{p}$. In particular, we show that the class $\mathcal{S}_{2} \circ \mathscr{L}$ coincides with the class of Cohen strongly 2summing multilinear operators. The same results are true for polynomial mappings. We end this paper by giving some factorization theorems concerning this new class.

We begin by recalling briefly some basic notations and terminology. Let $X$ be a Banach space; then $B_{X}$ is its closed unit ball and $X^{*}$ is its topological dual. Let now $m \in \mathbb{N}^{*}$ and let $X_{1}, \ldots, X_{m}, Y$ be Banach spaces over $\mathbb{K}(\mathbb{K}=\mathbb{R}$ or $\mathbb{C})$. We will denote by $\mathscr{L}\left(X_{1}, \ldots, X_{m} ; Y\right)$ the space of all continuous $m$-linear operators from $X_{1} \times \cdots \times X_{m}$ into $Y$. In the case $X_{1}=\cdots=X_{m}=X$, we will simply write $\mathscr{L}\left({ }^{m} X ; Y\right)$. As usual, $X_{1} \widehat{\otimes}_{\pi} \cdots \widehat{\otimes}_{\pi} X_{m}$ stands for the (complete) projective tensor product of the Banach spaces $X_{1}, \ldots, X_{m}$. If $T \in$ $\mathscr{L}\left(X_{1}, \ldots, X_{m} ; Y\right)$, we denote by $\widehat{T}$ the linearization of $T$, which is the linear map $\widehat{T}: X_{1} \widehat{\otimes}_{\pi} \cdots \widehat{\otimes}_{\pi} X_{m} \rightarrow Y$ given by $\widehat{T}\left(\sum_{i=1}^{n} x_{i}^{1} \otimes \cdots \otimes x_{i}^{m}\right)=\sum_{i=1}^{n} T\left(x_{i}^{1}, \ldots, x_{i}^{m}\right)$, for all $x_{i}^{j} \in X_{j}$ ( $n \in \mathbb{N}^{*}, 1 \leq i \leq n, 1 \leq j \leq m$ ). The canonical multilinear mapping $i_{m}: X_{1} \times \cdots \times X_{m} \rightarrow X_{1} \widehat{\otimes}_{\pi} \cdots \widehat{\otimes}_{\pi} X_{m}$ is defined 
by $i_{m}\left(x^{1}, \ldots, x^{m}\right)=x^{1} \otimes \cdots \otimes x^{m}$. We have the following factorization:

$$
T=\widehat{T} \circ i_{m}
$$

Let $T \in \mathscr{L}\left({ }^{m} X ; Y\right) ; T$ is symmetric if it is invariant for any permutation of its components. For any $T \in \mathscr{L}\left({ }^{m} X ; Y\right)$ we will denote by $T_{S}$ its associated symmetric multilinear operator, that is, $T_{S}$ defined by

$$
T_{S}\left(x^{1}, \ldots, x^{m}\right)=\frac{1}{m !} \sum_{\sigma} T\left(x^{\sigma(1)}, \ldots, x^{\sigma(m)}\right),
$$

where $\sigma$ is a permutation of the set $\{1, \ldots, m\}$. If $T$ is symmetric then $T_{S}=T$. A map $P: X \rightarrow Y$ is $m$-homogeneous polynomial if there exists a unique symmetric $m$-linear operator $\widehat{P}: X \times \stackrel{(m)}{\cdots} \times X \rightarrow Y$ such that $P(x)=\widehat{P}(x, \stackrel{(m)}{*}, x)$ for every $x \in X$. The polynomial $P$ is continuous if and only if $\widehat{P}$ is continuous. We denote, by $\mathscr{P}\left({ }^{m} X ; Y\right)$, the Banach space of all continuous $m$-homogeneous polynomials from $X$ to $Y$ endowed with the norm $\|P\|=\sup _{\|x\| \leq 1}\|P(x)\|$.

Let us recall the definitions of concept of $p$-summing $[5$, p. 31] and Cohen strongly $p$-summing operators [6], which will be used in the sequel.

Definition 1. Let $1 \leq p \leq \infty$ and $u: X \rightarrow Y$ be a linear operator.

(1) $u$ is $p$-summing if it takes weakly $p$-summable sequences in $X$ to strongly $p$-summable sequences in $Y$. We denote by $\Pi_{p}(X ; Y)$ the class of $p$-summing linear operators from $X$ to $Y$, which is a Banach space.

(2) $u$ is Cohen strongly $p$-summing if its adjoint operator $u^{*}: Y^{*} \rightarrow X^{*}$ is $p^{*}$-summing. We denote by $\mathscr{D}_{p}(X ; Y)$ the class of Cohen strongly $p$-summing linear operators from $X$ to $Y$, which is a Banach space.

If $K, H$ are Hilbert spaces, we have [6, Theorem 4.1.1]

$$
\mathcal{S}_{2}(K ; H)=\Pi_{p}(K ; H)=\mathscr{D}_{q}(K ; H),
$$

for $1 \leq p<\infty$ and $1<q \leq \infty$. If $1 \leq p \leq q<\infty$, we have

$$
\mathcal{S}_{p}(K ; H) \subset \mathcal{S}_{q}(K ; H) .
$$

Definition 2 (see [7]). Let $1 \leq p \leq \infty$. An $m$-linear operator $T: X_{1} \times \cdots \times X_{m} \rightarrow Y$ is Cohen strongly $p$-summing if its linearization $\widehat{T}$ is Cohen $p$-summing linear operator. The class of Cohen strongly $p$-summing $m$-linear operators from $X_{1} \times \cdots \times X_{m}$ to $Y$ is denoted by $\mathscr{D}_{p}^{m}\left(X_{1}, \ldots, X_{m} ; Y\right)$, which is a Banach space.

Definition 3 (see [8]). Let $1 \leq p \leq \infty$. $m$-homogeneous polynomial $P: X \rightarrow Y$ is Cohen strongly $p$-summing if its symmetric $m$-linear operator $\widehat{P}$ is Cohen strongly $p$-summing multilinear operator. The class of Cohen strongly $p$-summing $m$-homogeneous polynomials from $X$ to $Y$ is denoted by $\mathscr{P}_{\mathrm{Coh}}^{p}\left({ }^{m} X ; Y\right)$, which is a Banach space.

\section{Main Results}

2.1. Multilinear Mappings Generated by $\mathcal{S}_{p}$. Let $1 \leq p<\infty$. Let $X$ be a Banach space and $H$ a Hilbert space. The linear operator $u: X \rightarrow H$ is said to be of type $\delta_{p} \circ \mathscr{B}$, in symbols $u \in \mathcal{S}_{p} \circ \mathscr{B}(X ; H)$, if it factors through a Hilbert space $K$

$$
u: X \stackrel{u_{1}}{\longrightarrow} K \stackrel{u_{2}}{\longrightarrow} H
$$

where $u_{2} \in \mathcal{S}_{p}(K ; H)$. The space $\mathcal{S}_{p} \circ \mathscr{B}(X ; H)$ is a Banach space with the following norm:

$$
\|u\|_{\mathcal{S}_{p} \circ \mathscr{B}}=\inf \left\|u_{2}\right\|_{\mathcal{S}_{p}}\left\|u_{1}\right\|
$$

where the infimum is taken over all possible factorizations of the form (5). If $X$ is a Hilbert space, we have, for $1 \leq p<\infty$, $\mathcal{S}_{p} \circ \mathscr{B}(X ; H)=\mathcal{S}_{p}(X ; H)$.

Proposition 4. Let $X$ be a Banach space and $H$ a Hilbert space. Then

(1) if $1 \leq p \leq q<\infty$, we have $\mathcal{S}_{p} \circ \mathscr{B}(X ; H) \subset \mathcal{S}_{q}$ 。 $\mathscr{B}(X ; H)$;

(2) If $1<p<\infty$, we have

$$
u \in \mathcal{S}_{p} \circ \mathscr{B}(X ; H) \Longleftrightarrow u^{* *} \in \mathcal{S}_{p} \circ \mathscr{B}\left(X^{* *} ; H\right) .
$$

Proof. (1) The result follows from (4).

(2) Let $u \in \mathcal{S}_{p} \circ \mathscr{B}(X ; H)$; then $u: X \stackrel{u_{1}}{\longrightarrow} K \stackrel{u_{2}}{\longrightarrow} H$, with $u_{2} \in \mathcal{S}_{p}(K ; H)$. The second adjoint of $u$ is given by

$$
u^{* *}: X^{* *} \stackrel{u_{1}^{* *}}{\longrightarrow} K \stackrel{u_{2}^{* *}}{\longrightarrow} H
$$

then $u_{2}^{* *} \in \mathcal{S}_{p}(K ; H)$ and consequently $u^{* *} \in \mathcal{S}_{p} \circ \mathscr{B}\left(X^{* *}\right.$; $H)$.

Reciprocally, we use the elementary identity $u^{* *} \circ k_{X}=$ $k_{H} \circ u$, where $k_{X}: X \rightarrow X^{* *}$ is the isometric embedding defined by

$$
k_{X}(x)\left(x^{*}\right)=x^{*}(x)
$$

Then $u=k_{H}^{-1} \circ u^{* *} \circ k_{X}$, and consequently $u \in \mathcal{S}_{p} \circ \mathscr{B}(X ; H)$.

Before the presentation of the next result, we need the following lemma.

Lemma 5. Let $X, Y$ be Banach spaces such that $Y$ is reflexive. Let $u: X \rightarrow Y$ be a Cohen strongly 2-summing linear operator. Then, $u$ factors through a Hilbert space, that is, $\exists$ a Hilbert space $H$ and two linear operators $v_{1}, v_{2}$ such that

$$
u=v_{2} \circ v_{1}: X \stackrel{v_{1}}{\longrightarrow} H \stackrel{v_{2}}{\longrightarrow} Y \text {, }
$$

with $v_{2} \in \mathscr{D}_{2}(H ; Y)$.

Proof. Let $u \in \mathscr{D}_{2}(X ; Y)$. By [6, Theorem 2.2.2], its adjoint $u^{*}: Y^{*} \rightarrow X^{*}$ is 2 -summing. Then, $u^{*}$ factors through a Hilbert space $H$ (see the Pietsch Factorization Theorem 
[5, Corollary 2.16]); that is, $u^{*}=v_{2} \circ v_{1}: Y^{*} \stackrel{v_{1}}{\rightarrow} H \stackrel{v_{2}}{\rightarrow} X^{*}$, where $v_{1}$ is 2 -summing (i.e., its adjoint $v_{1}^{*}$ is Cohen strongly 2 -summing). The second adjoint of $u$ is given by

$$
u^{* *}=v_{1}^{*} \circ v_{2}^{*}: X^{* *} \stackrel{v_{2}^{*}}{\longrightarrow} H \stackrel{v_{1}^{*}}{\longrightarrow} Y^{* *} .
$$

On the other hand, we have $u^{* *} \circ k_{X}=k_{Y} \circ u$ with $k_{Y}$ being bijective ( $Y$ is reflexive). Consequently, we obtain $u=\left(k_{Y}^{-1}\right.$ 。 $\left.v_{1}^{*}\right) \circ\left(v_{2}^{*} \circ k_{X}\right)$. This completes the proof of Lemma 5 .

There is an interesting relationship between Cohen strongly $p$-summing linear operators and linear operators of type $\mathcal{S}_{2} \circ \mathscr{B}$.

Theorem 6. Let $X$ be a Banach space and $H$ a Hilbert space. For all $2 \leq p \leq \infty$, we have

$$
\mathcal{S}_{2} \circ \mathscr{B}(X ; H)=\mathscr{D}_{p}(X ; H)
$$

Proof. Let $u \in \mathcal{S}_{2} \circ \mathscr{B}(X ; H)$. By the factorization (5), $u=$ $u_{2} \circ u_{1}$ with $u_{2} \in \mathcal{S}_{2}(K ; H)$ and $u_{1} \in \mathscr{B}(X ; K)$. By (3) and the ideal property, we have $u \in \mathscr{D}_{p}(X ; H)$. Reciprocally, let $2 \leq p \leq \infty$ and $u \in \mathscr{D}_{p}(X ; H)$. By [6, Theorem 2.4.1], $u$ is in $\mathscr{D}_{2}(X ; H)$. According to Lemma $5, u$ factors through a Hilbert space

$$
u=u_{2} \circ u_{1}: X \stackrel{u_{1}}{\longrightarrow} K \stackrel{u_{2}}{\longrightarrow} H
$$

with $u_{2} \in \mathscr{D}_{2}(K ; H)\left(=\mathcal{S}_{2}(K ; H)\right)$. Therefore, $u=u_{2} \circ u_{1} \epsilon$ $\mathcal{S}_{2} \circ \mathscr{B}(X ; H)$.

Now, we introduce a similar definition to the category of multilinear mappings. A multilinear operator $T: X_{1} \times \cdots \times$ $X_{m} \rightarrow H$ is said to be of type $\mathcal{S}_{p} \circ \mathscr{L}$, in symbols $T \in \mathcal{S}_{p}$ 。 $\mathscr{L}\left(X_{1}, \ldots, X_{m}, H\right)$, if there exist a Hilbert space $K$, a linear operator $u \in \mathcal{S}_{p}(K ; H)$, and $A \in \mathscr{L}\left(X_{1}, \ldots, X_{m} ; K\right)$ such that

$$
T=u \circ A .
$$

The space $\mathcal{S}_{p} \circ \mathscr{L}\left(X_{1}, \ldots, X_{m} ; H\right)$ is a Banach space with the following norm:

$$
\|T\|_{\mathcal{S}_{p} \circ \mathscr{L}}=\inf \|u\|_{\mathcal{S}_{p}}\|A\|
$$

where the infimum is taken over all possible factorizations of form (14). Since $u$ is compact, the composed operator in (14) is compact by the properties of compact multilinear operators (see [4]), then every operator in the space $\mathcal{S}_{p}$ 。 $\mathscr{L}\left(X_{1}, \ldots, X_{m} ; H\right)$ is compact. Moreover, if $1 \leq p \leq q<\infty$ we have

$$
\mathcal{S}_{p} \circ \mathscr{L}\left(X_{1}, \ldots, X_{m} ; H\right) \subseteq \mathcal{S}_{q} \circ \mathscr{L}\left(X_{1}, \ldots, X_{m} ; H\right) .
$$

Our theorem below deals with the relation between a multilinear operator of type $\delta_{p} \circ \mathscr{L}$ and its linearization.

Theorem 7. Let $X_{1}, \ldots, X_{m}$ be Banach spaces and $H$ a Hilbert space. Let $T \in \mathscr{L}\left(X_{1}, \ldots, X_{m} ; H\right)$. The following properties are equivalent.
(1) The multilinear operator $T$ belongs to $\mathcal{S}_{p} \circ \mathscr{L}\left(X_{1}\right.$, $\left.\ldots, X_{m} ; H\right)$.

(2) The linearization $\widehat{T} \in \mathcal{S}_{p} \circ \mathscr{B}\left(X_{1} \widehat{\otimes}_{\pi} \cdots \widehat{\otimes}_{\pi} X_{m} ; H\right)$.

Proof. First, we suppose that $T$ is of type $\delta_{p} \circ \mathscr{L}$. Then, by the factorization (14) we have $T=u \circ A$ with $u \in \mathcal{S}_{p}(K ; H)$. So, by using (1) we obtain $\widehat{T}=u \circ \widehat{A}$, where $\widehat{A}$ is the linearization of $A$; then

$$
\widehat{T} \in \mathcal{S}_{p} \circ \mathscr{B}\left(X_{1} \widehat{\otimes}_{\pi} \cdots \widehat{\otimes}_{\pi} X_{m} ; H\right) .
$$

Now, we suppose that the second assertion is true. We can write $T=\widehat{T} \circ i_{m}=u_{2} \circ u_{1} \circ i_{m}$, with $u_{2} \in \mathcal{S}_{p}(K ; H)$.

As in the linear case (12), we can establish the relation between Cohen strongly $p$-summing multilinear operators and multilinear operators of type $\delta_{2} \circ \mathscr{L}$.

Theorem 8. Let $X_{1}, \ldots, X_{m}$ be Banach spaces and $H$ a Hilbert space. For all $2 \leq p \leq \infty$, we have

$$
\mathcal{S}_{2} \circ \mathscr{L}\left(X_{1}, \ldots, X_{m} ; H\right)=\mathscr{D}_{p}^{m}\left(X_{1}, \ldots, X_{m} ; H\right) .
$$

Proof. Let $T \in \mathcal{S}_{2} \circ \mathscr{L}\left(X_{1}, \ldots, X_{m} ; H\right)$. By the factorization (14), $T=u \circ A$ with $u \in \mathcal{S}_{2}(K ; H)$ and $A \in \mathscr{L}\left(X_{1}, \ldots, X_{m} ; K\right)$. By (3) and [9, Corollary 4.2], we have

$$
u \circ A \in \mathscr{D}_{p}^{m}\left(X_{1}, \ldots, X_{m} ; H\right) \text {. }
$$

Reciprocally, let $2 \leq p \leq \infty$ and $T \in \mathscr{D}_{p}^{m}\left(X_{1}, \ldots, X_{m} ; H\right)$. By [7, Corollary 2.5], $T$ is in $\mathscr{D}_{2}^{m}\left(X_{1}, \ldots, X_{m} ; H\right)$ and, by [9, Corollary 4.2], we have $T=u \circ A$, where $u \in \mathscr{D}_{2}(Z ; H)$ and $Z$ is a Banach space. According to Lemma 5, $u$ factors through a Hilbert space; that is, $u=u_{2} \circ u_{1}$ with $u_{2} \in \mathscr{D}_{2}(K ; H)$ $\left(=\mathcal{S}_{2}(K ; H)\right)$. Therefore,

$$
T=u_{2} \circ u_{1} \circ A \in \mathcal{S}_{2} \circ \mathscr{L}\left(X_{1}, \ldots, X_{m} ; H\right) ;
$$

the desired result follows.

Theorem 9. Let $H_{1}, \ldots, H_{m}, H$ be Hilbert spaces. Then,

(1) if $2<p<\infty$, we have $\mathscr{D}_{2}^{m}\left(X_{1}, \ldots, X_{m}\right.$; $\left.H\right) \subset \mathcal{S}_{p}$ 。 $\mathscr{L}\left(X_{1}, \ldots, X_{m} ; H\right)$;

(2) if $1 \leq p<2$, we have $\mathcal{S}_{p} \circ \mathscr{L}\left(X_{1}, \ldots, X_{m} ; H\right) \subset$ $\mathscr{D}_{2}^{m}\left(X_{1}, \ldots, X_{m} ; H\right)$.

Proof. (1) Let $2<p<\infty$ and $T \in \mathscr{D}_{2}^{m}\left(X_{1}, \ldots, X_{m} ; H\right)$. By (18), $T$ belongs to $\mathcal{S}_{2} \circ \mathscr{L}\left(X_{1}, \ldots, X_{m} ; H\right)$ and the result follows by (16).

(2) Let $1 \leq p<2$ and $T$ be a multilinear operator of type $\mathcal{S}_{p} \circ \mathscr{L}$. Then, $T=u \circ A$, with $u \in \mathcal{S}_{p}(K ; H)$. It follows by (3) that $u \in \mathscr{D}_{2}(K ; H)$. Thus [9, Corollary 4.2] completes the proof of Theorem 9 .

2.2. Factorization of Schatten Class Type Mappings. In the linear case, it is well known that Hilbert Schmidt operators factor through $\mathscr{L}_{1}$-space or $\mathscr{L}_{\infty}$-space (Lindenstrauss and Pelczynski [10]) and also through infinite dimensional 
Banach spaces [5, Theorem 19.2]. The converse is also true in both cases. For the multilinear and polynomial cases, every Hilbert Schmidt multilinears or polynomial mappings factor through any Banach spaces, but the converse is not true (see [11, Theorem 2.10. and Example 2.12]). In this section, we consider the particular class $\mathcal{S}_{2} \circ \mathscr{L}$ for which it is possible to obtain an extension similar to the linear case cited above.

First, we recall the definition of $p$-factorable multilinear operators introduced by Cerna Maguina in [12] as a generalization of the one given by Diestel et al. in the linear case [5].

Definition 10. Let $1 \leq p \leq \infty$ and $X_{1}, \ldots, X_{m}, Y$ be Banach spaces. The operator $T: X_{1} \times \cdots \times X_{m} \rightarrow Y$ is said to be $p$-factorable if there exist a measure space $(\Omega, \Sigma, \mu), u \in$ $\mathscr{B}\left(L_{p}(\mu) ; Y^{* *}\right)$, and $B \in \mathscr{L}\left(X_{1}, \ldots, X_{m} ; L_{p}(\mu)\right)$ such that

$$
K_{Y} \circ T=u \circ B,
$$

where $K_{Y}$ is the isometric embedding of $Y$ into $Y^{* *}$. We denote by $\mathscr{L}_{p \text {-fat }}\left(X_{1}, \ldots, X_{m} ; Y\right)$ the space of all $p$-factorable multilinear operators, which is a Banach space.

Theorem 11 (see [12, Proposition 2.3]). Let $X_{1}, \ldots, X_{m}, Y$ be Banach spaces. Then, the following assertions are equivalent.

(1) The operator $T$ belongs to $\mathscr{L}_{2-f a t}\left(X_{1}, \ldots, X_{m} ; Y\right)$.

(2) The operator $T$ factors through a Hilbert space; that is, there exist a Hilbert space $H, u \in \mathscr{B}(H ; Y)$, and $B \in$ $\mathscr{L}\left(X_{1}, \ldots, X_{m} ; H\right)$ such that $T=u \circ B$.

We now present a factorization result for mappings of Schatten class type $\mathcal{S}_{2} \circ \mathscr{L}$.

Theorem 12. Let $X_{1}, \ldots, X_{m}$ be Banach spaces and $H a$ Hilbert space. Then, the following properties are equivalent.

(1) The operator $T \in \mathcal{S}_{2} \circ \mathscr{L}\left(X_{1}, \ldots, X_{m} ; H\right)$.

(2) There exist a linear operator $u \in \mathscr{B}\left(\mathscr{L}_{1} ; H\right)$ and $B \in$ $\mathscr{L}_{2-\text { fat }}\left(X_{1}, \ldots, X_{m} ; \mathscr{L}_{1}\right)$ such that $T=u \circ B$.

(3) There exist $u \in \mathscr{B}\left(\mathscr{L}_{\infty} ; H\right)$ and $B \in \mathscr{L}_{2 \text {-fat }}\left(X_{1}, \ldots\right.$, $\left.X_{m} ; \mathscr{L}_{\infty}\right)$ such that $T=u \circ B$.

Proof. (1) $\Rightarrow$ (2): Let $T=u \circ A$ with $u \in \mathcal{S}_{2}(K ; H)$. The operator $u$ factors through $\mathscr{L}_{1}$-space; that is, $u=v_{1} \circ v_{2}$ with $v_{2} \in \mathscr{B}\left(K ; \mathscr{L}_{1}\right)$ and $v_{1} \in \mathscr{B}\left(\mathscr{L}_{1} ; H\right)$; thus

$$
T: X_{1} \times \cdots \times X_{m} \stackrel{A}{\rightarrow} K \stackrel{v_{2}}{\rightarrow} \mathscr{L}_{1} \stackrel{v_{1}}{\rightarrow} H
$$

that is, $T=v_{1} \circ B$ where $B=v_{2} \circ A \in \mathscr{L}_{2 \text {-fat }}\left(X_{1}, \ldots, X_{m} ; \mathscr{L}_{1}\right)$.

(2) $\Rightarrow$ (1): Let $T=u \circ B$ with $u \in \mathscr{B}\left(\mathscr{L}_{1} ; H\right)$ and $B \in$ $\mathscr{L}_{2 \text {-fat }}\left(X_{1}, \ldots, X_{m} ; \mathscr{L}_{1}\right)$. One can write $B$ as

$$
B=v \circ A: X_{1} \times \cdots \times X_{m} \stackrel{A}{\rightarrow} K_{1} \stackrel{v}{\rightarrow} \mathscr{L}_{1} .
$$

By Grothendieck's Theorem [5, Theorem 3.1], the operator $u$ is 2-summing. So it factors through a Hilbert space; that is, $u=s_{1} \circ s_{2}: \mathscr{L}_{1} \stackrel{s_{2}}{\rightarrow} K_{2} \stackrel{s_{1}}{\rightarrow} H$ with $s_{2} \in \Pi_{2}\left(\mathscr{L}_{1} ; K_{2}\right)$. Therefore, $T=u \circ B=s_{1} \circ s_{2} \circ v \circ A$ with $s_{1} \circ s_{2} \circ v \in \mathcal{S}_{2}\left(K_{1} ; H\right)$; this ends the proof.

(3) $\Leftrightarrow(1)$ : This is a similar argument to the last one.
Now, we give a multilinear version of the Diestel-JarchowTonge result. The proof is similar to that of the last Theorem. So that we omit its proof.

Theorem 13. Let $X_{1}, \ldots, X_{m}$ be Banach spaces and $H a$ Hilbert space. The following properties are equivalent.

(1) The operator $T \in \mathcal{S}_{2} \circ \mathscr{L}\left(X_{1}, \ldots, X_{m} ; H\right)$.

(2) For every Banach space $Z$, there exist $u \in \mathscr{B}(Z ; H)$ and $B \in \mathscr{L}_{2-f a t}\left(X_{1}, \ldots, X_{m} ; Z\right)$ such that $T=u \circ B$.

To close this paper, we introduce the defintion of polynomial of type $\mathcal{S}_{p} \circ \mathscr{P}$. A polynomial mapping $P \in \mathscr{P}\left({ }^{m} \mathrm{X} ; H\right)$ is said to be of type $\mathcal{S}_{p} \circ \mathscr{P}$, in symbols $P \in \mathcal{S}_{p} \circ \mathscr{P}\left({ }^{m} X ; H\right)$, if its $m$-linear symmetric $\widehat{P}$ is of type $\mathcal{S}_{p} \circ \mathscr{L}$. The space $\mathcal{S}_{p} \circ \mathscr{P}\left({ }^{m} X, H\right)$ is a Banach space with the following norm: $\|P\|_{\mathcal{S}_{p} \circ \mathscr{P}}=\|\widehat{P}\|_{\mathcal{S}_{p} \circ \mathscr{L}}$. By applying similar argument of that given for the multilinear case, we have the following result.

Theorem 14. Let $X$ be a Banach space and $H$ a Hilbert space. Then

(1) if $2 \leq p<\infty$, we have $\mathscr{P}_{\mathrm{Coh}}^{2}\left({ }^{m} X ; H\right) \subseteq \mathcal{S}_{p}$ 。 $\mathscr{P}\left({ }^{m} X ; H\right)$;

(2) if $1 \leq p \leq 2$, we have $\mathcal{S}_{p} \circ \mathscr{P}\left({ }^{m} X ; H\right) \subseteq \mathscr{P}_{\mathrm{Coh}}^{2}\left({ }^{m} X ; H\right)$.

Proof. (1) If $P \in \mathscr{P}_{\mathrm{Coh}}^{2}\left({ }^{m} X ; H\right)$ then its $m$-linear symmetric $\widehat{P}$ belongs to $\mathscr{D}_{2}^{m}\left({ }^{m} X ; Y\right)$. By Theorems 8 and $9, \widehat{P} \in \mathcal{S}_{p}$ 。 $\mathscr{L}\left({ }^{m} X ; H\right)$, and thus $P \in \mathcal{S}_{p} \circ \mathscr{P}\left({ }^{m} X ; H\right)$.

(2) Let $P \in \mathcal{S}_{p} \circ \mathscr{P}\left({ }^{m} X ; H\right)$; then $\widehat{P}$ is of type $\mathcal{S}_{p} \circ \mathscr{L}$. The result follows from Theorems 8 and 9 .

As consequence of the last Theorems, we have the following corollary.

Corollary 15. Let $X$ be a Banach space and $H$ a Hilbert space. The following properties are equivalent.

(1) The polynomial $P \in \mathcal{S}_{2} \circ \mathscr{P}\left({ }^{m} X ; H\right)$.

(2) There exist a linear operator $u \in \mathscr{B}\left(\mathscr{L}_{\infty} ; H\right)$ and $Q \in \mathscr{P}\left({ }^{m} X ; \mathscr{L}_{\infty}\right)$ such that $P=u \circ Q$ with $\widehat{Q} \in$ $\mathscr{L}_{2-f a t}\left({ }^{m} X ; \mathscr{L}_{\infty}\right)$.

(3) There exist a linear operator $u \in \mathscr{B}\left(\mathscr{L}_{1} ; H\right)$ and $Q \in \mathscr{P}\left({ }^{m} X ; \mathscr{L}_{1}\right)$ such that $P=u \circ Q$ with $\widehat{Q} \in$ $\mathscr{L}_{2-f a t}\left({ }^{m} X ; \mathscr{L}_{1}\right)$.

(4) For every Banach space $Z$, there exist $u \in \mathscr{B}(Z ; H)$ and $Q \in \mathscr{P}\left({ }^{m} X ; Z\right)$ such that $P=u \circ Q$ with $\widehat{Q} \in$ $\mathscr{L}_{2-f a t}\left({ }^{m} X ; Z\right)$.

Proof. (1) $\Leftrightarrow$ (2): If $P \in \mathcal{S}_{2} \circ \mathscr{P}\left({ }^{m} X ; H\right)$ then $\widehat{P} \in$ $\mathcal{S}_{2} \circ \mathscr{L}\left({ }^{m} X ; H\right)$. So, by Theorem $12, \widehat{P}$ factors through $\mathscr{L}_{\infty^{-}}$ space; that is, $\widehat{P}=u \circ B$, with $u \in \mathscr{B}\left(\mathscr{L}_{\infty} ; H\right)$ and $B \in$ $\mathscr{L}_{2 \text {-fat }}\left({ }^{m} X ; \mathscr{L}_{\infty}\right)$. Now, it is not difficult to show that $\widehat{P}_{S}=$ $u \circ B_{S}$ and $B_{S} \in \mathscr{L}_{2 \text {-fat }}\left({ }^{m} X ; \mathscr{L}_{\infty}\right)$. Then $P=u \circ Q$ where $\widehat{Q}=B_{S}$. 
Reciprocally, we suppose that the second assertion is true. We have $\widehat{P}=u \circ \widehat{Q}$; by Theorem $12 \widehat{P}$ belongs to $\mathcal{S}_{2}$ 。 $\mathscr{L}\left({ }^{m} X ; H\right)$; that is, $P \in \mathcal{S}_{2} \circ \mathscr{P}\left({ }^{m} X ; H\right)$.

(1) $\Leftrightarrow(3)$ and (1) $\Leftrightarrow(4)$ : The rest are similar to the last one.

\section{Competing Interests}

The authors declare that they have no competing interests.

\section{References}

[1] A. Pietsch, "Ideals of multilinear functionals (designs of a theory)," in Proceedings of the 2nd International Conference on Operator Algebras, Ideals and their Applications in Theoretical Physics, pp. 185-199, Teubner-Texte, Leipzig, Germany, 1983.

[2] H.-A. Braunss and H. Junek, "On types of polynomials and holomorphic functions on Banach spaces," Note di Matematica, vol. 10, no. 1, pp. 47-58, 1990.

[3] C. de Andrade Mendes, "On factorization of Schatten class type mappings," Mathematische Nachrichten, vol. 279, no. 9-10, pp. 988-995, 2006.

[4] G. Botelho, D. Pellegrino, and P. Rueda, "On composition ideals of multilinear mappings and homogeneous polynomials," Publications of the Research Institute for Mathematical Sciences, Kyoto University, vol. 43, no. 4, pp. 1139-1155, 2007.

[5] J. Diestel, H. Jarchow, and A. Tonge, Absolutely Summing Operators, vol. 43 of Cambridge Studies in Advanced Mathematics, Cambridge University Press, 1995.

[6] J. S. Cohen, "Absolutely $p$-summing, $p$-nuclear operators and their conjugates," Mathematische Annalen, vol. 201, pp. 177-200, 1973.

[7] D. Achour and L. Mezrag, "On the Cohen strongly p-summing multilinear operators," Journal of Mathematical Analysis and Applications, vol. 327, no. 1, pp. 550-563, 2007.

[8] D. Achour and K. Saadi, "A polynomial characterization of Hilbert spaces," Collectanea Mathematica, vol. 61, no. 3, pp. 291301, 2010.

[9] L. Mezrag and K. Saadi, "Inclusion and coincidence properties for Cohen strongly summing multilinear operators," Collectanea Mathematica, vol. 64, no. 3, pp. 395-408, 2013.

[10] J. Lindenstrauss and A. Pelczynski, "Absolutely summing operators in $\ell_{p}$-spaces and their applications," Studia Mathematica, vol. 29, no. 3, pp. 275-326, 1968.

[11] C. A. Mendes, "On factorization of Hilbert-Schmidt mappings," Journal of Mathematical Analysis and Applications, vol. 304, no. 1, pp. 137-146, 2005.

[12] B. M. Cerna Maguina, Operadores multilineares p-fatoraveis [Ph.D. thesis], Universidade Estadual de Campinas, São Paulo, Brazil, 2005. 


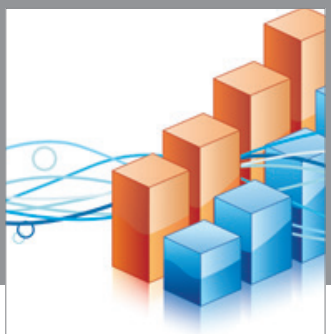

Advances in

Operations Research

vatem alat4

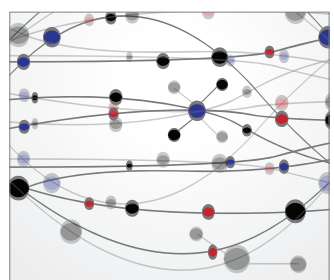

\section{The Scientific} World Journal
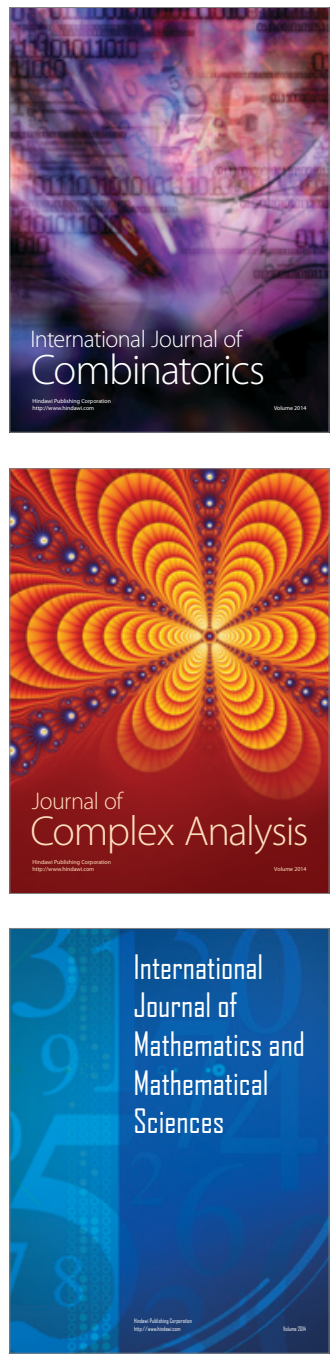
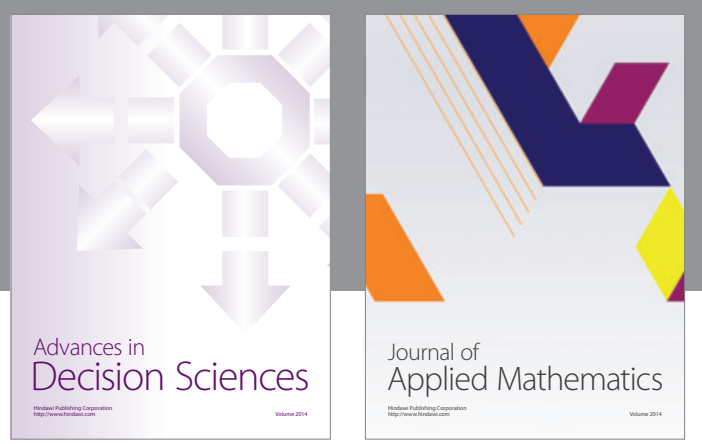

Algebra

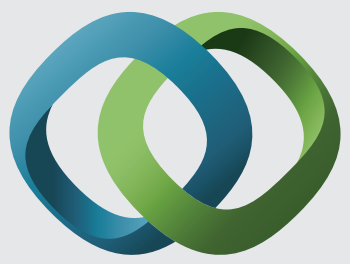

\section{Hindawi}

Submit your manuscripts at

http://www.hindawi.com
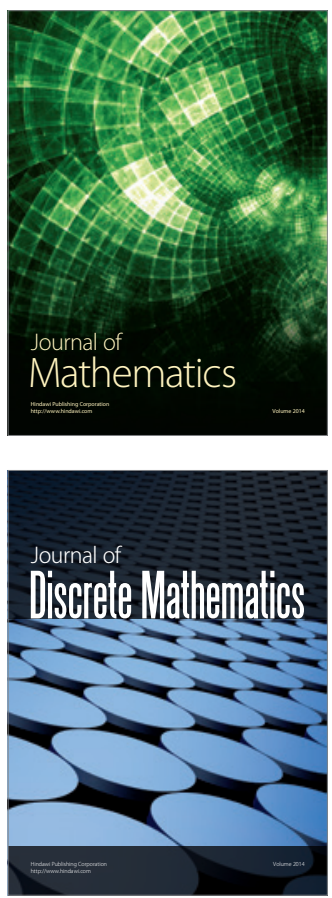

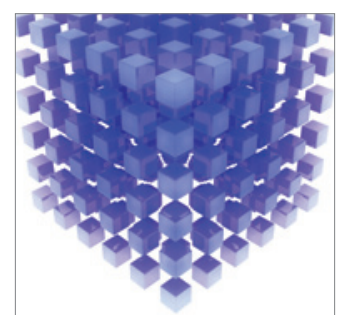

Mathematical Problems in Engineering
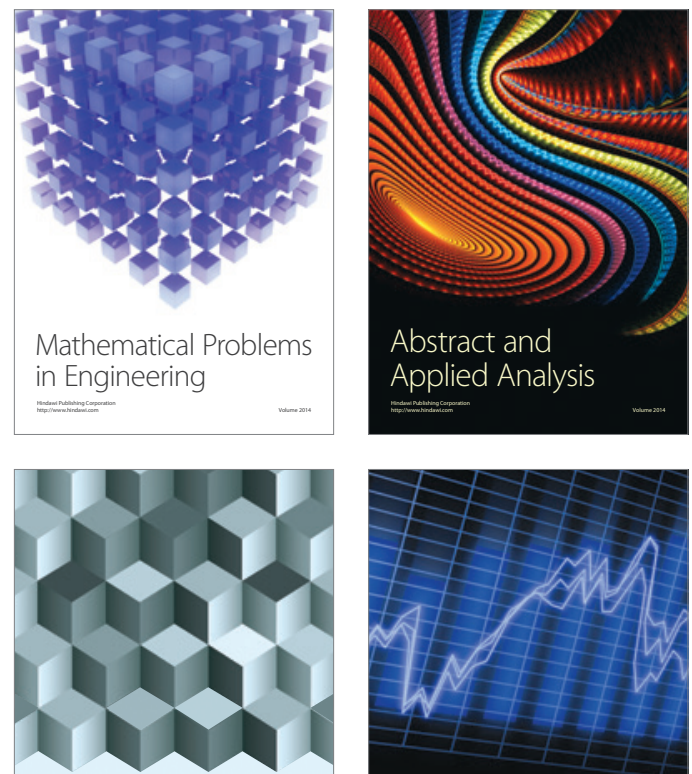

Journal of

Function Spaces

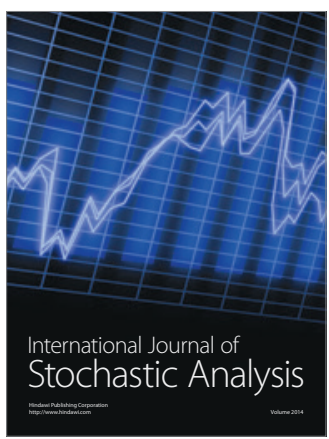

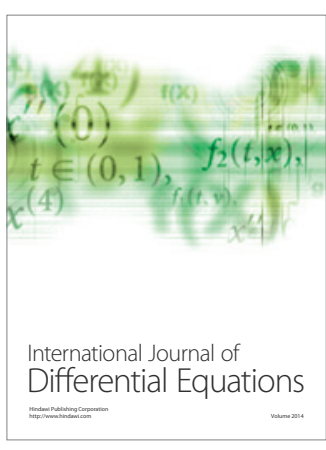
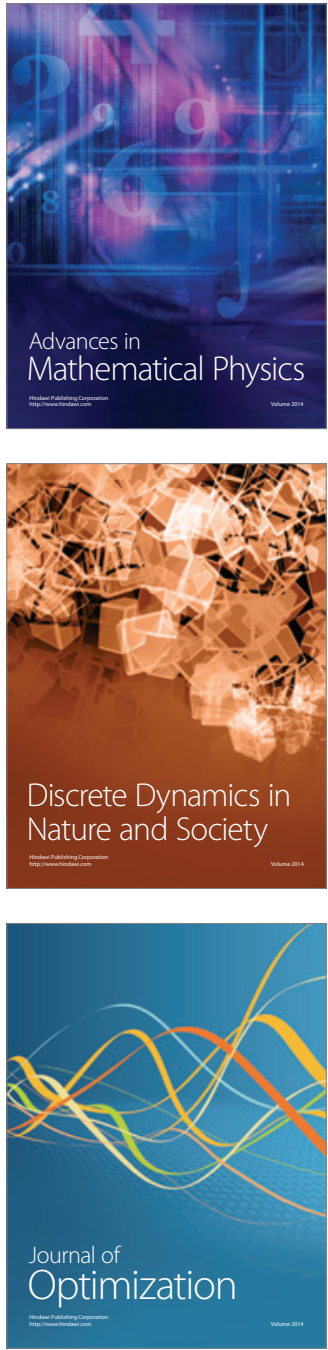